\title{
Efficient First-Principles Calculation of Phonon-Assisted Photocurrent in Large-Scale Solar-Cell Devices
}

\author{
Mattias Palsgaard, ${ }^{1,2, *}$ Troels Markussen, ${ }^{1}$ Tue Gunst, ${ }^{2}$ Mads Brandbyge, ${ }^{2}$ and Kurt Stokbro ${ }^{1}$ \\ ${ }^{1}$ Synopsys-QuantumWise A/S, Fruebjergvej 3, Postbox 4, DK-2100 Copenhagen, Denmark \\ ${ }^{2}$ Department of Micro- and Nanotechnology (DTU Nanotech), Center for Nanostructured Graphene (CNG), \\ Technical University of Denmark, DK-2800 Kgs. Lyngby, Denmark
}

(Received 21 December 2017; revised manuscript received 20 April 2018; published 25 July 2018)

\begin{abstract}
We present a straightforward and computationally cheap method to obtain the phonon-assisted photocurrent in large-scale devices from first-principles transport calculations. The photocurrent is calculated using the nonequilibrium Green's function with light-matter interaction from the first-order Born approximation, while electron-phonon coupling (EPC) is included through special thermal displacements. We apply the method to a silicon solar-cell device and demonstrate the impact of including EPC in order to properly describe the current due to the indirect band-to-band transitions. The first-principles results are successfully compared to experimental measurements of the temperature and light-intensity dependence of the open-circuit voltage of a silicon photovoltaic module. Our calculations illustrate the pivotal role played by EPC in photocurrent modeling to avoid underestimation of the open-circuit voltage, shortcircuit current, and maximum power. This work represents a recipe for computational characterization of future photovoltaic devices including the combined effects of light-matter interaction, phonon-assisted tunneling, and the device potential at finite bias from the level of first-principles simulations.
\end{abstract}

DOI: 10.1103/PhysRevApplied.10.014026

\section{INTRODUCTION}

Photovoltaics (PV) represents a promising technology as a replacement for the burning of fossil fuels. In the past couple of decades, many promising thin-film absorber materials have been discovered, all of them with unique strengths and weaknesses. CdTe and copper indium gallium selenide [CIGS, $\mathrm{CuIn}_{(1-x)} \mathrm{Ga}_{(x)} \mathrm{Se}_{2}$ ] can produce high efficiencies, but include rare and toxic elements, while copper zinc tin sulfide (CZTS, $\mathrm{Cu}_{2} \mathrm{ZnSnS}_{4}$ ) includes only nontoxic earth-abundant elements, but suffers from low efficiency and open-circuit voltage $\left(V_{\mathrm{OC}}\right)$ [1-4]. Clearly, there is still room for the discovery of new materials to improve on the cost-efficiency relationship. The field of computational material science has seen massive progression, and as a result the difference between the system size and complexity attainable in simulations and experiments is becoming smaller every day. Recently, a review was published on the design of new materials using firstprinciples calculations [5]. Here, it is stressed how the

\footnotetext{
*mlnp@nanotech.dtu.dk
}

Published by the American Physical Society under the terms of the Creative Commons Attribution 4.0 International license. Further distribution of this work must maintain attribution to the author(s) and the published article's title, journal citation, and DOI. abundance of candidate materials together with the lack of efficient devices highlight the need for efficient predictive device calculations. Continuum models are used extensively in the field of PV to extract benchmark parameters from measurements on devices and to predict the performance of new device geometries [6]. It is difficult to include important effects such as confinement of electrons and phonons, surface effects, and strain in the continuum models. These effects can, however, be captured using atomistic models based on density-functional theory (DFT). DFT combined with the nonequilibrium Green's function (NEGF) formalism [7-9] has, for example, previously been used to improve a continuum-model study of transport through the interface between CZTS and the buffer material CdS, which is important for CZTS solar-cell efficiency $[10,11]$. In spite of the influx of new thin-film-based PV cells, silicon remains the market leader and about $90 \%$ of PV cells are still based on silicon, where large modules with high efficiency and stability can be produced [1].

Silicon has an indirect band gap and as such, in order to conserve momentum, absorption of a photon below the fundamental direct gap energy must be accompanied by the absorption or emission of a phonon. A number of recent studies also show that EPC plays a key role in the outstanding performance of PV cells based on direct-band-gap perovskites [12-15]. 
The study of phonon-assisted photon absorption from first principles is notoriously difficult as it involves a double sum over fine grids of $\mathbf{k}$ points and complex two-excitation processes. Therefore state-of-the-art DFT calculations of phonon-assisted absorption have so far been limited to bulk crystals where the supercell contains only a few atoms [16-18], while empirical models have been used for studies of realistic devices [19]. Recently, Zacharias and Giustino [18] introduced a very efficient method for including phonon-induced absorption processes using a single supercell calculation in which the atoms are displaced away from their equilibrium positions. We recently adopted this special-thermal-displacement (STD) approach to study electron transport in silicon systems with over 1000 atoms, including electron-phonon coupling within the DFT NEGF formalism [20].

In this paper we apply STD to calculations of the firstorder photocurrent in a 19.6-nm silicon $p-n$ junction from DFT NEGF. In this way we are able to capture the phononassisted absorption over the indirect band gap of silicon and study directly the effect of temperature on the performance of the device. In the following section, we summarize the methodology used to calculate the photocurrent using the first-order Born approximation and the inclusion of EPC through the STD approach. Exhaustive derivations of the important equations can be found in previous publications [18,20-23].

\section{METHOD}

We calculate the photocurrent as a first-order perturbation to the electronic system caused by the interaction with a weak electromagnetic field. The electron-photon interaction is given by the Hamiltonian

$$
H^{\prime}=\frac{e}{m_{0}} \mathbf{A} \cdot \mathbf{P},
$$

where $\mathbf{A}$ is the vector potential and $\mathbf{P}$ is the momentum operator. For a single-mode monochromatic light source, we have [21]:

$$
\mathbf{A}=\mathbf{e}\left(\frac{\hbar \sqrt{\tilde{\mu}_{r} \tilde{\epsilon}_{r}}}{2 N \omega \tilde{\epsilon} c} F\right)^{1 / 2}\left(b e^{-i \omega t}+b^{\dagger} e^{i \omega t}\right)
$$

where $\tilde{\mu}_{r}$ is the relative permeability, $\tilde{\epsilon}_{r}$ is the relative permittivity, $\tilde{\epsilon}$ is the permittivity, $\omega$ is the frequency of the light, $F$ is the photon flux, $N$ is the number of photons, $b^{\dagger}$ and $b$ are the bosonic creation and annihilation operators, and $\mathbf{e}$ is a unit vector giving the polarization of the light.

Using the standard Meir-Wingreen formula [24] and including only first-order terms in $F$, we arrive at an expression similar to Fermi's golden rule for the current into the left $(L)$ and right $(R)$ leads due to absorption of photons $[23,25]$ :

$$
\begin{aligned}
& I_{\alpha}= \frac{e}{h} \int_{-\infty}^{\infty} \sum_{\beta=L, R}\left[1-f_{\alpha}(E)\right] f_{\beta}(E-\hbar \omega) T_{\alpha, \beta}^{-}(E) \\
&-f_{\alpha}(E)\left[1-f_{\beta}(E+\hbar \omega)\right] T_{\alpha, \beta}^{+}(E) d E, \\
& T_{\alpha, \beta}^{-}(E)=N \operatorname{Tr}\left\{M^{\dagger} \tilde{A}_{\alpha}(E) M A_{\beta}(E-\hbar \omega)\right\}, \\
& T_{\alpha, \beta}^{+}(E)=N \operatorname{Tr}\left\{M \tilde{A}_{\alpha}(E) M^{\dagger} A_{\beta}(E+\hbar \omega)\right\},
\end{aligned}
$$

where $\alpha \in L, R, f_{\alpha}$ is the Fermi-Dirac distribution function of lead $\alpha, A_{\alpha}=G \Gamma_{\alpha} G^{\dagger}$ is the spectral function of lead $\alpha$, $\tilde{A}_{\alpha}=G^{\dagger} \Gamma_{\alpha} G$ is the time-reversed spectral function of lead $\alpha$, and the electron-photon coupling matrix is

$$
M_{m l}=\frac{e}{m_{0}}\left(\frac{\hbar \sqrt{\tilde{\mu}_{r} \tilde{\epsilon}_{r}}}{2 N \omega \tilde{\epsilon} c} F\right)^{1 / 2} \mathbf{e} \cdot \mathbf{P}_{m l} .
$$

The total photocurrent is then calculated as $I_{\mathrm{ph}}=I_{L}-I_{R}$. The retarded $G$ and advanced $G^{\dagger}$ Green's functions, the spectral broadening of the leads $\Gamma_{\alpha}$, and the momentum operator $\mathbf{P}$ are calculated self-consistently from DFT NEGF simulations of the silicon $p-n$ junction device.

The temperature-dependent EPC is included through a single displacement of the atomic positions according to $[18,20]$

$$
\mathbf{u}_{\mathrm{STD}}(T)=\sum_{\lambda}(-1)^{\lambda-1} \sigma_{\lambda}(T) \mathbf{e}_{\lambda},
$$

where $\mathbf{e}_{\lambda}$ is the eigenvector of phonon mode $\lambda$ and the Gaussian width $\sigma$ is given by

$$
\sigma_{\lambda}(T)=l_{\lambda} \sqrt{2 n_{\lambda}(T)+1},
$$

where $n_{\lambda}(T)$ and $l_{\lambda}=\sqrt{\hbar / 2 M_{p} \Omega_{\lambda}}$ are the Bose-Einstein occupation and vibrational characteristic length of mode $\lambda$ with frequency $\Omega_{\lambda}$. Phonon modes are obtained using a supercell method [26-28]. The configuration displaced according to Eq. (7) gives the correct thermal average of the Landauer conductance and the optical absorption for sufficiently large systems with many repetitions of the same unit cell $[18,20]$.

In summary the total current at bias $V$ and temperature $T$ is calculated as follows:

$$
I(V, T)=I_{0}\left[V, \mathbf{u}_{\mathrm{STD}}(T)\right]+I_{\mathrm{ph}}\left[V, \mathbf{u}_{\mathrm{STD}}(T)\right]
$$

where $I_{0}$ is the dark current coming from the applied bias. By displacing the atomic coordinates once according 


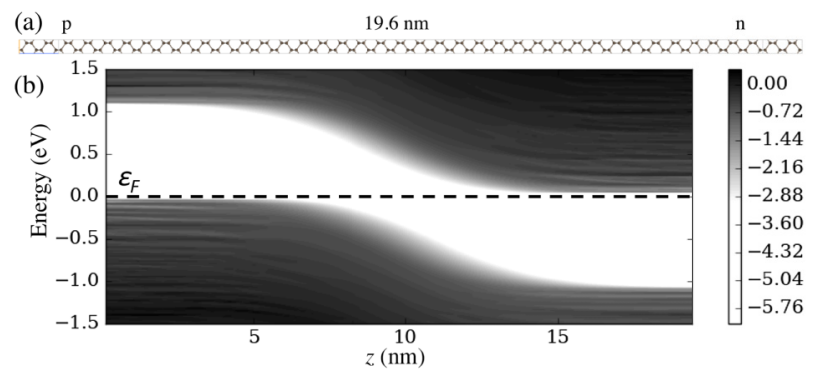

FIG. 1. (a) Structure and cell used in the calculation of the 19.6-nm silicon $p$ - $n$ junction, with $n=2 \times 10^{19} \mathrm{~cm}^{-3}$. (b) Local density of states along the transport direction of the silicon $p-n$ junction on a logarithmic scale.

to Eq. (7), calculating, and adding the two current contributions, we get the current under illumination of a single frequency of light. To calculate the total current under sunlight illumination, we integrate the current over frequency with the flux $F$ given by the AM1.5 reference spectrum. All DFT NEGF calculations in this study were performed using the ATK DFT software [29-31], with the SG15-low basis set and $11 \times 11(21 \times 21) \mathbf{k}$ points in the electronic structure (transport) calculations. DFT within the local-density approximation (LDA) or the generalizedgradient approximation (GGA) to the exchange-correlation potential is known to underestimate the band gaps in semiconductors. In order to overcome this band-gap problem, we used the GGA+1/2 exchange-correlation method [32], which yields accurate band structures for a wide range of materials with the same computational effort as normal GGA calculations. With these parameters we obtain an indirect band gap of $1.135 \mathrm{eV}$ for pristine silicon (neglecting zero-point motion). All phonon calculations were performed using Tersoff potentials $[33,34]$. Electronic doping was included by adding a charge fixed at the position of every silicon atom in the structure, as in previous work [35]. The DFT electronic structure under applied bias was calculated self-consistently using the standard NEGF approach [31].

\section{RESULTS AND DISCUSSION}

Figure 1(a) shows the considered 19.6-nm silicon $p$ $n$ junction with transport along the [100] direction. The related local density of states is shown in Fig. 1(b) and we can see the typical $p$ - $n$ profile along the device, with flat bands near the electrode indicating converged screening potentials [20,35]. Furthermore, we see that the calculated band gap is very close to the one observed in experiments on silicon [36].

In Fig. 2(a) we compare the photocurrent density calculated for a pristine silicon (neglecting zero-point motion) $p-n$ junction with that of a $300-\mathrm{K}$ STD structure for $F=1 / \AA^{2}$ s. The dashed lines indicate the energies of the indirect $\left(E_{g}^{\text {indirect }}=1.135 \mathrm{eV}\right)$ and direct $\left(E_{g}^{\text {direct }}=2.853\right.$ $\mathrm{eV}$ ) band gaps of the bulk silicon structure using the same calculational settings. First, we see that the obtained band gap agrees well with the experimental value (1.12 eV) [36], verifying our use of the GGA+1/2 exchangecorrelation method. Second, we see that the inclusion of EPC through the STD results in an increase in photocurrent from the indirect transition of about two orders of magnitude. The photocurrent as a function of photon energy at $300 \mathrm{~K}$ compares well to previous calculations of the absorption coefficients in bulk silicon, where EPC was included in the same way [18]. Temperature effects on the electronic structure are included in the STD method, and indeed we see a finite photocurrent at photon energies below the bulk band gap corresponding to a reduction in the band gap with temperature. In addition to the finitetemperature renormalization of the band gap, we obtain the actual photocurrent of the transport setup assisted by band-to-band tunneling and including the device potential at finite bias. Unlike calculations on bulk silicon, we see here a small finite contribution to the photocurrent coming from the indirect transition even without EPC. This we trace back to symmetry breaking by the ultrathin $p-n$ junction and the resulting device potential along the [100] direction. This current contribution is analyzed in more detail in the Supplemental Material [37]. In simulations based on the bulk-silicon band structure, such transitions
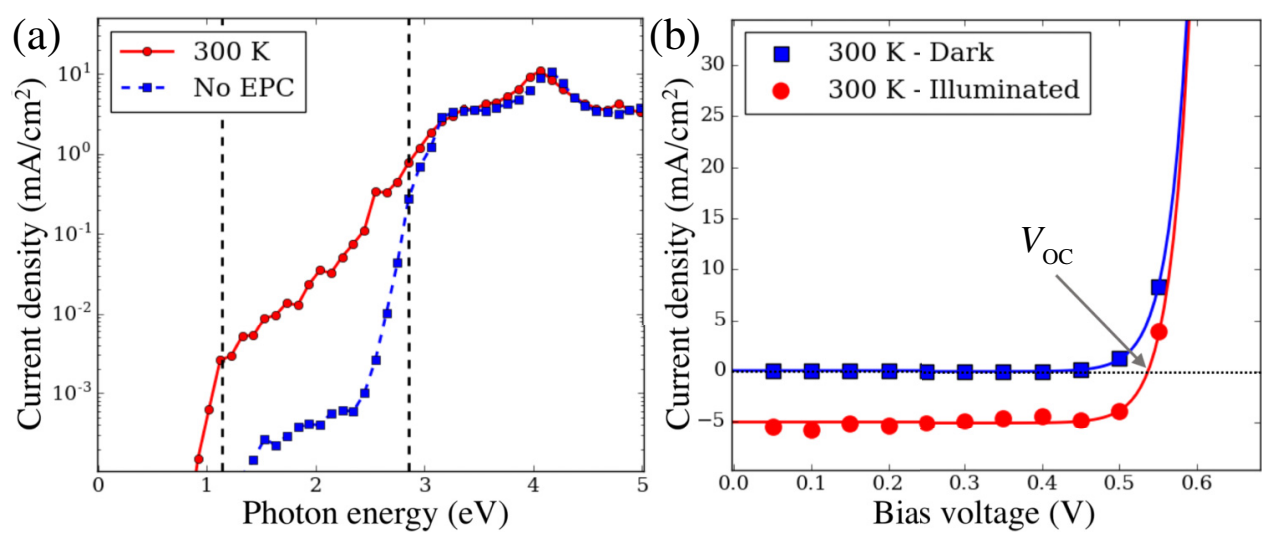

FIG. 2. (a) Calculated photocurrent density for the pristine (blue dashed) and STD displaced (red solid) system, where $F=1 / \AA^{2}$ s and $V=50 \mathrm{mV}$. (b) Calculated room-temperature current density as a function of voltage for the silicon $p$ - $n$ junction in the dark $(F=$ 0 ) and under illumination. 

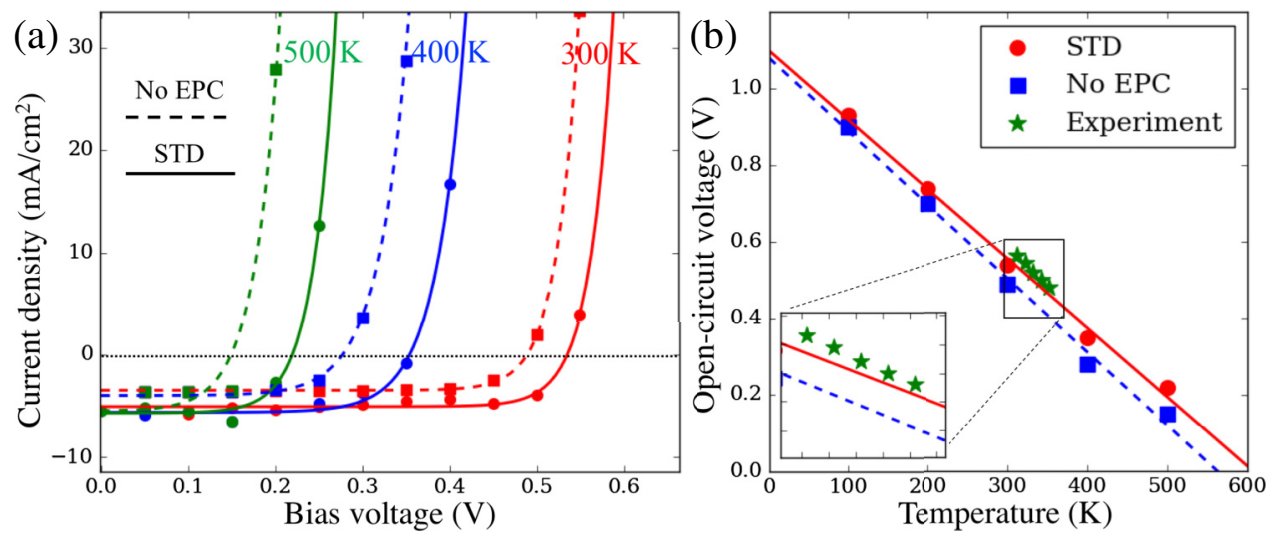

FIG. 3. (a) Calculated $I-V$ curves with (solid) and without (dashed) EPC for different temperatures. (b) Calculated open-circuit voltage as a function of temperature with linear fits. The green star markers are experimental measurements from [40]. The inset shows a close-up near the experimental measurements. In all calculations and experimental values shown in this figure, a light intensity of 1 sun was used.

would be prohibited by the opposite symmetry of conductance and valence states if not assisted by phonons. This follows from the selection rule due to momentum conservation, which is a consequence of translational symmetry. However, in a $p$ - $n$ junction, the system is not translationally invariant in the transport direction, and hence the momentum in the transport direction is not a conserved quantity. The effects of phonon-assisted tunneling, temperature renormalization, and the device potential are all seen to play an important role in the quantitative photocurrent device characteristics. Regarding below-gap transitions, a similar result was seen previously when studying theoretically the phonon-assisted tunneling into graphene in a scanning-tunneling-spectroscopy setup [38]. Here a finite but strongly suppressed tunneling into the Dirac point of graphene is seen even below the threshold voltage of the phonon opening the inelastic channel. In order to generate an $I-V$ curve, we need to calculate the total photocurrent under sunlight illumination. To that end we used the AM1.5 reference spectrum and integrated the spectral current densities for a certain applied bias.

The $I-V$ curve of the silicon $p-n$ junction is shown in Fig. 2(b), with and without the addition of photocurrent. A least-squares fit of the calculated data points to the usual expression for the current of a diode under illumination $I=I_{\mathrm{ph}}+I_{0}\left[\exp \left(q V / n k_{B} T\right)-1\right]$ is added. The result looks very much like what is expected [39], with a photocurrent being a nearly constant contribution at all applied biases. The applied bias voltage where the illuminated $I-V$ curve crosses the zero-current-density line and no current is generated is known as the open-circuit voltage $\left(V_{\mathrm{OC}}\right)$ and is a measurable parameter used to benchmark solar-cell performance. For crystalline-silicon PV cells, the open-circuit voltage is known to be in the range $0.55-0.60 \mathrm{~V}$ [40-42] at room temperature, in good agreement with the $0.54 \mathrm{~V}$ obtained from our calculation. The short-circuit current, obtained at zero applied bias, varies a lot depending on the quality and device geometry of the measured solar cell, making a direct comparison with our results difficult. The obtained $5 \mathrm{~mA} \mathrm{~cm}^{-2}$ is, however, aligned with typical values published in the literature $[41,42]$.

To analyze the direct impact of EPC on the device, we plot in Fig. 3(a) the $I-V$ curve under illumination for the perfectly symmetric (noninteracting) silicon system together with that of the system where atomic positions are displaced according to STD [Eq. (7)] for different temperatures. Without EPC the short-circuit current density is underestimated by about $25 \%$. Importantly, the open-circuit voltage is significantly underestimated without EPC and one obtains $V_{\mathrm{OC}}=0.49 \mathrm{~V}$ at $300 \mathrm{~K}$. The open-circuit voltage of a PV device is an important performance indicator that defines its quality and is typically very well controlled, so a $\sim 10 \%$ loss at room temperature
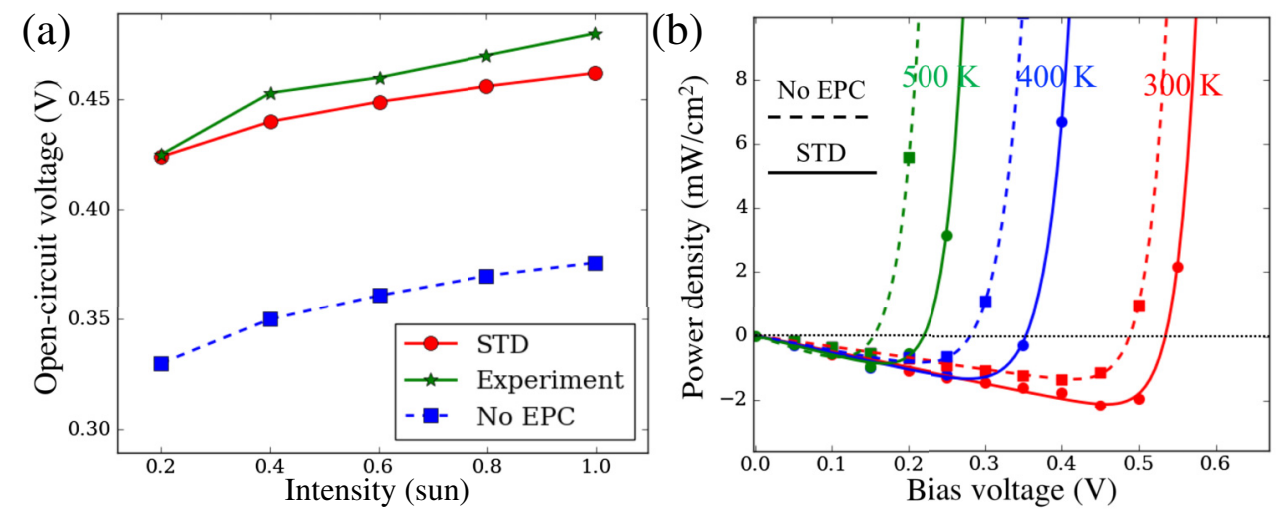

FIG. 4. (a) Open-circuit voltage as a function of light intensity at $352 \mathrm{~K}$. The calculated results including EPC were extrapolated from the fitted line in Fig. 3(b), since we did not perform calculations at the exact temperature measured in [40]. (b) Calculated power density as a function of applied voltage with (solid) and without (dashed) EPC for different temperatures. 
is substantial. This highlights the importance of including phonon effects in device calculations of indirect semiconductor PV devices.

We will now analyze the temperature dependence of the device characteristics, which can often be extracted from experiments. The short-circuit current is largely constant while the open-circuit voltage is degraded for higher temperatures, due to the increased probability of carriers tunneling through the $p-n$ junction via electron-phonon scattering events at bias voltages below the band gap. In the case with no EPC, the increased current at higher temperatures comes only from the temperature dependence of the Fermi-Dirac distribution. Comparing with the results where EPC is ignored (dashed lines), we see that the opencircuit voltage is systematically underestimated and that the error is larger at high temperature. It is not surprising that the inclusion of EPC is more important at higher temperatures where the phonon population is higher. The short-circuit current is also constant for the case without EPC, but it is too low at all temperatures. Using the results shown in Fig. 3(a), we can extract the opencircuit voltage, which is often measured in experiments on solar-cell devices, as a function of temperature. The open-circuit voltage as a function of temperature with and without the inclusion of EPC is shown in Fig. 3(b). A linear fit was performed on both data sets using leastsquares fitting, and the best fit was plotted alongside the data points. For both cases, we get the expected linear temperature dependence. The open-circuit voltage extrapolated to the $T=0 \mathrm{~K}$ point is often used in experiments to extract the activation energy of the dominant recombination path. Here we get $1.1 \mathrm{~V} \pm 0.025 \mathrm{~V}$ with EPC and $1.08 \mathrm{~V} \pm 0.029 \mathrm{~V}$ without EPC, both slightly below our calculated band gap. In Fig. 3(b) we also compare the calculated results with experiments carried out by Huang et al. [40], where the open-circuit voltage of a crystallinesilicon PV module was measured under simulated solar irradiation while controlling the cell temperature. The calculated results including EPC agree nicely with the experimental measurements. The calculated open-circuit voltages without the inclusion of EPC are much lower than the experimental values at all temperatures. Experimental measurements of $V_{\mathrm{OC}}$ performed at temperatures in the range 100-300 $\mathrm{K}$ under 1.1 sun illumination [41] also agree nicely with the values calculated including EPC (not shown).

Lastly, we will analyze the trends with light intensity. In Fig. 4(a) we show the calculated open-circuit voltage for different intensities of the light source at a temperature of $352 \mathrm{~K}$ and compare with experimental values from Huang et al. [40]. Again we see that the results where EPC is included through STD agree nicely with experimental measurements. The best agreement is seen at 0.2 sun, which is to be expected given the assumption of a weak field going into Eq. (1). On the other hand, the open-circuit voltages calculated while neglecting EPC do not agree with the experimental values. In fact, even the results calculated at 1 sun are below the experimental values, where only an intensity of 0.2 sun is used. This underlines the pivotal role played by EPC in PV devices. Figure 4(b) shows the generated power density for the $p-n$ junction with and without the inclusion of EPC. We see that the maximum power and maximum power point are both underestimated in the case without EPC.

\section{CONCLUSION}

We have presented a computationally cheap method to calculate the phonon-assisted photocurrent in large-scale devices from first principles. Previous studies of phononassisted optical absorption using state-of-the-art methods have been limited to bulk systems of high symmetry, where only a handful of atoms are considered due to the computational cost. Here we study a 19.6-nm-long silicon $p$ - $n$ junction under working conditions with an applied bias. The calculated current density as a function of photon energy is similar in shape to the temperature-renormalized bulk optical absorption of silicon calculated in previous studies using a similar description of EPC [18]. Our results agree nicely with experiments both for values of the open-circuit voltages and for trends in how they scale, with temperature and light intensity. The phonon interaction has a significant impact on the device characteristics, highlighting the need for photocurrent transport calculations including phonon coupling when considering devices using indirect semiconductors as the absorber material. The combination of device simulations with photon coupling as well as phonon coupling, through special thermal displacements, offers an appealing way forward in the difficult problems of combined light-matter interaction, phonon-assisted tunneling, temperature renormalization, and the nonequilibrium device potential in quantitative first-principles simulations.

\section{ACKNOWLEDGMENTS}

This work is partly funded by the Innovation Fund Denmark (IFD) under File No. 5016-00102. We thank Andrea Crovetto and Urs Aeberhard for their input on this work.

[1] S. Philipps and W. Warmuth, Photovoltaics Report, Tech. Rep. (Fraunhofer Institute for Solar Energy Systems, ISE, 2017).

[2] V. M. Fthenakis, Life cycle impact analysis of cadmium in CdTe PV production, Renew. Sustain. Energy Rev. 8, 303 (2004).

[3] M. Woodhouse, A. Goodrich, R. Margolis, T. James, R. Dhere, T. Gessert, T. Barnes, R. Eggert, and D. Albin, Perspectives on the pathways for cadmium telluride photovoltaic module manufacturers to address expected increases 
in the price for tellurium, Sol. Energy Mater. Sol. Cells 115, 199 (2013).

[4] A. Polman, M. Knight, E. C. Garnett, B. Ehrler, and W. C. Sinke, Photovoltaic materials: Present efficiencies and future challenges, Science 352, aad4424 (2016).

[5] K. T. Butler, J. M. Frost, J. M. Skelton, K. L. Svane, and A. Walsh, Computational materials design of crystalline solids, Chem. Soc. Rev. 45, 6138 (2016).

[6] M. Burgelman, P. Nollet, and S. Degrave, Modelling polycrystalline semiconductor solar cells, Thin Solid Films 361-362, 527 (2000).

[7] L. V. Keldysh, Diagram technique for nonequilibrium processes, Sov. Phys. JETP 20, 1018 (1965).

[8] R. A. Craig, Perturbation expansion for real-time Green's functions, J. Math. Phys. 9, 605 (1968).

[9] P. Danielewicz, Quantum theory of nonequilibrium processes, I, Ann. Phys. 152, 239 (1984).

[10] A. Crovetto, M. L. N. Palsgaard, T. Gunst, T. Markussen, K. Stokbro, M. Brandbyge, and O. Hansen, Interface band gap narrowing behind open circuit voltage losses in $\mathrm{Cu}_{2} \mathrm{ZnSnS}_{4}$ solar cells, Appl. Phys. Lett. 110, 083903 (2017).

[11] M. L. N. Palsgaard, A. Crovetto, T. Gunst, T. Markussen, O. Hansen, K. Stokbro, and M. Brandbyge, Semiconductor band alignment from first principles: A new nonequilibrium Green's function method applied to the CZTSe/CdS interface for photovoltaics, in SISPAD 2016 (2016), pp. 377-380.

[12] J. Yang, X. Wen, H. Xia, R. Sheng, Q. Ma, J. Kim, P. Tapping, T. Harada, T. W. Kee, F. Huang, Y.-B. Cheng, M. Green, A. Ho-Baillie, S. Huang, S. Shrestha, R. Patterson, and G. Conibeer, Acoustic-optical phonon up-conversion and hot-phonon bottleneck in lead-halide perovskites, Nat. Commun. 8, 14120 (2017).

[13] H. Kim, J. Hunger, E. Cánovas, M. Karakus, Z. Mics, M. Grechko, D. Turchinovich, S. H. Parekh, and M. Bonn, Direct observation of mode-specific phonon-band gap coupling in methylammonium lead halide perovskites, Nat. Commun. 8, 687 (2017).

[14] A. D. Wright, C. Verdi, R. L. Milot, G. E. Eperon, M. A. Pérez-Osorio, H. J. Snaith, F. Giustino, M. B. Johnston, L. M. Herz, Electron-phonon coupling in hybrid lead halide perovskites, Nat. Commun. 7, 11755 (2016).

[15] P.-A. Mante, C. C. Stoumpos, M. G. Kanatzidis, and A. Yartsev, Electron-acoustic phonon coupling in single crystal $\mathrm{CH}_{3} \mathrm{NH}_{3} \mathrm{PbI}_{3}$ perovskites revealed by coherent acoustic phonons, Nat. Commun. 8, 14398 (2017).

[16] J. Noffsinger, E. Kioupakis, C. G. Van de Walle, S. G. Louie, and M. L. Cohen, Phonon-Assisted Optical Absorption in Silicon from First Principles, Phys. Rev. Lett. 108, 167402 (2012).

[17] M. Zacharias, C. E. Patrick, and F. Giustino, Stochastic Approach to Phonon-Assisted Optical Absorption, Phys. Rev. Lett. 115, 177401 (2015).

[18] M. Zacharias and F. Giustino, One-shot calculation of temperature-dependent optical spectra and phonon-induced band-gap renormalization, Phys. Rev. B 94, 075125 (2016).

[19] U. Aeberhard, Quantum-kinetic theory of photocurrent generation via direct and phonon-mediated optical transitions, Phys. Rev. B 84, 035454 (2011).
[20] T. Gunst, T. Markussen, M. L. N. Palsgaard, K. Stokbro, and M. Brandbyge, First-principles electron transport with phonon coupling: Large scale at low cost, Phys. Rev. B 96, 161404 (2017).

[21] L. E. Henrickson, Nonequilibrium photocurrent modeling in resonant tunneling photodetectors, J. Appl. Phys. 91, 6273 (2002).

[22] L. Zhang, K. Gong, J. Chen, L. Liu, Y. Zhu, D. Xiao, and H. Guo, Generation and transport of valley-polarized current in transition-metal dichalcogenides, Phys. Rev. B 90, 195428 (2014).

[23] J. Chen, Y. Hu, and H. Guo, First-principles analysis of photocurrent in graphene $p n$ junctions, Phys. Rev. B 85, 155441 (2012).

[24] Y. Meir and N. S. Wingreen, Landauer Formula for the Current Through an Interacting Electron Region, Phys. Rev. Lett. 68, 2512 (1992).

[25] C. Rivas, R. Lake, G. Klimeck, W. R. Frensley, M. V. Fischetti, P. E. Thompson, S. L. Rommel, and P. R. Berger, Full-band simulation of indirect phonon assisted tunneling in a silicon tunnel diode with delta-doped contacts, Appl. Phys. Lett. 78, 814 (2001).

[26] T. Gunst, T. Markussen, K. Stokbro, and M. Brandbyge, First-principles method for electron-phonon coupling and electron mobility: Applications to two-dimensional materials, Phys. Rev. B 93, 035414 (2016).

[27] G. J. Ackland, M. C. Warren, and S. J. Clark, Practical methods in ab initio lattice dynamics, J. Phys. Condens. Matter 9, 7861 (1997).

[28] K. Kunc and R. M. Martin, Ab initio Force Constants of GaAs: A New Approach to Calculation of Phonons and Dielectric Properties, Phys. Rev. Lett. 48, 406 (1982).

[29] Atomistix ToolKit version 2017.1, Synopsys-QuantumWise A/S.

[30] J. M. Soler, E. Artacho, J. D. Gale, A. García, J. Junquera, P. Ordejón, and D. Sánchez-Portal, The SIESTA method for $a b$ initio order $-N$ materials simulation, J. Phys.: Condens. Matter 14, 2745 (2002).

[31] M. Brandbyge, J.-L. Mozos, P. Ordejón, J. Taylor, and K. Stokbro, Density-functional method for nonequilibrium electron transport, Phys. Rev. B 65, 165401 (2002).

[32] L. G. Ferreira, M. Marques, and L. K. Teles, Approximation to density functional theory for the calculation of band gaps of semiconductors, Phys. Rev. B 78, 125116 (2008).

[33] J. Tersoff, Empirical interatomic potential for silicon with improved elastic properties, Phys. Rev. B 38, 9902 (1988).

[34] J. Schneider, J. Hamaekers, S. T. Chill, S. Smidstrup, J. Bulin, R. Thesen, A. Blom, and K. Stokbro, ATKforcefield: A new generation molecular dynamics software package, Modell. Simul. Mater. Sci. Eng. 25, 085007 (2017).

[35] D. Stradi, U. Martinez, A. Blom, M. Brandbyge, and K. Stokbro, General atomistic approach for modeling metalsemiconductor interfaces using density functional theory and nonequilibrium Green's function, Phys. Rev. B 93, 155302 (2016).

[36] M. Levinshtein, S. Rumyantsev, and M. Shur, Handbook Series on Semiconductor Parameters (World Scientific, Singapore, 1996), Vol. 1. 
[37] See Supplemental Material at http://link.aps.org/supplemen tal/10.1103/PhysRevApplied.10.014026 for [convergence of the STD photocurrent with $p-n$ junction size and an extended discussion about photocurrent contributions coming from photons with energies below the direct band gap of silicon.].

[38] M. L. N. Palsgaard, N. P. Andersen, and M. Brandbyge, Unravelling the role of inelastic tunneling into pristine and defected graphene, Phys. Rev. B 91, 121403 (2015).

[39] P. Würfel, Physics Today (Wiley-VCH Verlag GmbH \& Co. KGaA, Weinheim, 2005).
[40] B. J. Huang, P. E. Yang, Y. P. Lin, B. Y. Lin, H. J. Chen, R. C. Lai, and J. S. Cheng, Solar cell junction temperature measurement of PV module, Sol. Energy 85, 388 (2011).

[41] P. Löper, D. Pysch, A. Richter, M. Hermle, S. Janz, M. Zacharias, and S. W. Glunz, Analysis of the temperature dependence of the open-circuit voltage, Energy Procedia 27, 135 (2012).

[42] S. Chander, A. Purohit, A. Sharma, S. P. Nehra, and M. S. Dhaka, A study on photovoltaic parameters of monocrystalline silicon solar cell with cell temperature, Energy Rep. 1, 104 (2015). 